\title{
HBIM METHODOLOGY AS A BRIDGE BETWEEN ITALY AND ARGENTINA
}

\author{
A. Moreira ${ }^{\text {a }}$, R. Quattrini ${ }^{b}, *$, G. Maggiolo ${ }^{\text {a }}$, R. Mammoli ${ }^{\text {b }}$ \\ ${ }^{a}$ Faculty of Architecture, Design and Urbanism, National University of the Litoral, Santa FE, Argentina \\ amoreira@fadu.unl.edu.ar, gastonmaggiolo@gmail.com \\ ${ }^{\mathrm{b}}$ DICEA, Dept. of Civil and Building Engineering and Architecture, Polytechnic University of Marche, Ancona, Italy \\ r.quattrini@univpm.it,r.mammoli@pm.univpm.it
}

Commission II, WG II/8

KEY WORDS: Semantic Modelling, Built Memory, Parametric Intelligence, Reality Based Modelling, Ontologies

\begin{abstract}
:
The availability of efficient HBIM workflows could represent a very important change towards a more efficient management of the historical real estate. The present work shows how to obtain accurate and reliable information of heritage buildings through reality capture and 3D modelling to support restoration purposes or knowledge-based applications. Two cases studies metaphorically joint Italy with Argentina. The research article explains the workflows applied at the Palazzo Ferretti at Ancona and the Manzana Histórica de la Universidad National del Litoral, providing a constructive comparison and blending technological and theoretical approaches. In a bottom-up process, the assessment of two cases study validates a workflow allowing the achievement of a useful and proper data enrichment of each HBIM model. Another key aspect is the Level of Development (LOD) evaluation of both models: different ranges and scales are defined in America (100-500) and in Italy (A-G), nevertheless is possible to obtain standard shared procedures, enabling facilitation of HBIM development and diffusion in operating workflows.
\end{abstract}

\section{INTRODUCTION}

The historical heritage in South America, as well as in Europe, is now demanding robust procedures for obtaining Heritage Building Information Modelling (HBIM) fully interoperable and rich in its informative content. The availability of efficient workflows for engineers and architects could represent a very important change towards a more efficient management of the historical real estate. The present work shows how to obtain accurate and reliable information of heritage buildings through reality capture and $3 \mathrm{D}$ modelling to support restoration purposes or knowledge-based application.

Today, two cases of study metaphorically joint Italy with Argentina. The present research article explains the workflows applied at the Palazzo Ferretti at Ancona and the Manzana Histórica de la Universidad National del Litoral (MHUNL), providing a constructive comparison from which it is possible to generalize a robust method for HBIM data management. In last years, several researches have been able to develop methods demonstrating the feasibility of a whole Building Information Modelling (BIM) approach for complex architectural shapes starting from point clouds. As result of this implementations, interesting developments arise when it is possible to work in 3D environment along the process to minimize the steps and format changes during the modelling and enrichment phases in order to avoid simplification or loss information. In this line, a focus is to achieve a good procedure able to overcome the challenge of creating BIM models to be used in cultural heritage.

Beginning from $2 \mathrm{D}$ drawings and $3 \mathrm{D}$ point cloud at high resolution, HBIM methodology could become a bridge of technology transfer that allows a complete management of future architectural and restoration project.
Thus, the strength of our work is the blending between a technological and a theoretical approach with reciprocal benefits. As in Argentina the lack of technological instruments is a limitation, the need to wisely fill the gap with alternative sources and conceive a scalable HBIM approach is mandatory. In order to achieve that, it seems appropriate to work under the realm of technology transfer as a system that defines and integrates purposes and practices. As well as, to work in a manner that provides the necessary tools to promote, capture and disseminate the intellectual capital (information) of an entire organization, enabling the classification and dissemination of tacit and explicit knowhow for the development of projects. One of the most relevant characteristics that demand its inclusion in the practice of HBIM as a link between knowledge and needs is historicathat it allows to make a parallel between the knowledge of "know how to do things" and "why we do things".

Traditionally, all the information related to heritage buildings has been represented by a collection of individual documents: original and non-original, official and unofficial drawings and surveys, bi-dimensional representations either hand or computer assisted drawings. These datasets were generated by different professionals, following their own methodologies, personal investigations, tools and standards. This information is dispersed in various locations meaning that there are miles between the documentation and the object, resulting in a total disconnection between the information, the building and the people responsible for its operation and management. We must also highlight the status and quality of this information, which is generally unknown; it may have been replaced, uncoordinated, or incomplete. In most cases, in Argentina but not only, there is no single source of reliable and consistent information about a heritage asset in particular.

\footnotetext{
* Corresponding author
} 


\section{STATE OF ART}

Recently, BIM and its related digital innovations have demonstrated to be a catalyst for more transparency, tighter integration, and increased productivity in the architecture, engineering, and construction industry (AEC). This technology transfer should be exploited and scaled up as a digital disruption (Merschbrock and Munkvold, 2015). Technology transfer does not refer to a connection of a machine with other machines and has nothing to do with a specific technology -it is not a computer or a laser beam. The concept involves the "transformation of available socio-technological resources, such as skills and tools, into solutions" (Schreyer, 2001). It should be noted that the acquisition of technologies does not necessarily lead to the acquisition of knowledge, nor the technological capacity to operate it. Instead, it provides dynamic knowledge, which generates a combined structure between knowledge and the infrastructure that makes possible to cope with any type of conditionings. This joining extends the scope of the process for the development of projects, covering legal terms, techniques, management and manufacture (Davenport and Prusak, 2000).

The difference between BIM and HBIM derives mainly from their goal: it is clear that HBIM as design tool should be an informative system able to define the state of conservation and planning future operations, along all the life-cycle of architectural heritage. More in general, literature shows differences both from the process point of view both from the conceptual scheme. Major challenges in HBIM diffusion are in the need of extending processes for as-planned building to asbuilt models (Volk et al., 2014). In addition, HBIM approach needs to face theoretical and pratical challenges and to fill gap regard the BIM: a wide discussion about differences between a) semantic object or unsegmented geometries, b) standardization or irregularity c) parametric intelligence or geometric accuracy is provided in (Apollonio et al., 2016). In HBIM field, the modelling phase and the semantic sub-division in various elements are sufficiently robust, although time-consuming (Garagnani and Manferdini, 2013) (Barazzetti et al., 2015) and based on proprietary tools .

According several studies, the observed trend in the definition of HBIM is the creation of a model starting from survey data (i.e. Terrestrial Laser Scanning (TLS), digital photogrammetry etc.), aimed at the creation of a library of parametrical objects, combined with the informative data in order to enrich the object besides the modelling phase (Quattrini et al., 2017). Moreover, highlighting the difference between modelling environment and real informed approach, a major challenge nowadays is to obtain a rich model usable in specific context. The final product of the HBIM process should be a model that, before being used as a professional tool, must be populated by non-geometrical data for making it suitable for engineering and conservation applications. The LOD is not only the graphical quality of the element but also the awareness about its characteristics, following the AIA definition and protocols ("https://www.aiacontracts.org/contract-documents/19016project-bim-protocol," n.d.). The UNI committee partially updated these definitions, overcoming the GRADE by the LOG. In (COMMITTE, 2017) it is significant the introduction of a specific LOD scale (F-G) for restoration.

The majority of the approaches provides desktop solutions, but the adoption of web-based and 3D-centered informative system have to be mentioned as challenging and promising development. Indeed these tools have several advantages; for example: several accesses by users from different locations at the same time; central storage for the whole documentation; no need of local application using WebGL graphic libraries; mapping the information with great accuracy on 3D models; complex queries to retrieve all of the information easily (Apollonio et al., 2018).

\section{TWO PIPELINES IN COMPARISON}

Considering previous highlighted advances in HBIM modelling, but also the lack of solutions to be extended to all cases and purposes of interventions, the paper shows two similar and complementary approaches with the goal of building up a solid workflow for reality-based models sufficiently rich of information and semantically structured. Weak and strong points of two workflows are also discussed and authors provide a comparison allowing to establish a concrete path in HBIM field.

\subsection{The Palazzo Ferretti's case study}

In regards to Palazzo Ferretti, the procedure allows to build a parametric model of this building in a Revit environment integrating several sources: Terrestrial Laser Scanner (TLS) survey served as main reference and for the correction of previous two-dimensional surveys, on the other hand photogrammetric survey was carried out for detailed information, in comparison with treatises recommendations (Figure1). A global information management provides a HBIM fully interoperable and with sufficient descriptive information for both the building and the intended use of this one, i.e. a museum. Today, in fact, the Palace is the location of National Archaeological Museum of Marche Region.

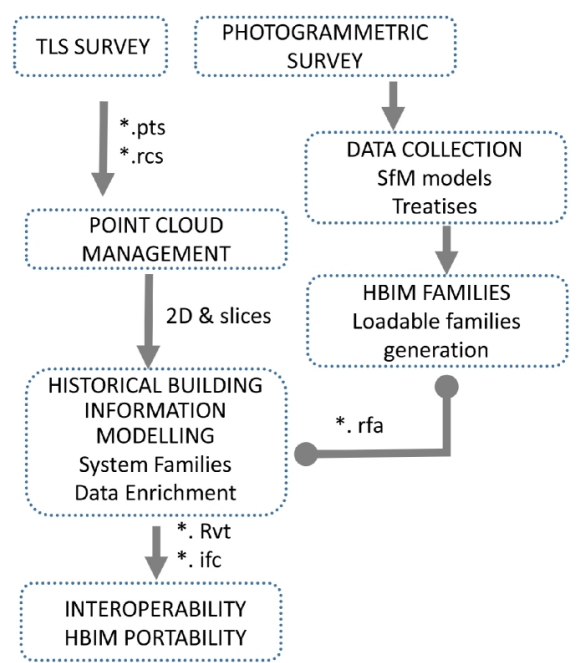

Figure 1. General workflow used for Palazzo Ferretti

Palazzo Ferretti was built in 1560 by Pellegrino Tibaldi with drawings provided by the great architect Antonio da Sangallo il Giovane. In a first step, we assumed to follow the same modelling procedure applied to the previous cases (Quattrini et al., 2015): this is a 3D cloud environment managed without proprietary licenses for the point cloud formats. However, for the entire extension of the building we were forced to use twodimensional drawings. Despite the considerable amount of data collected, the TLS campaign did not cover all wings of the Palace: 49 stations from the outside of the building and 20 stations from inside. The modelling in Revit environment highlighted some complexity especially in the case of not perfectly orthogonal walls and in the need to define a several vertical reference planes (Figure 2). 


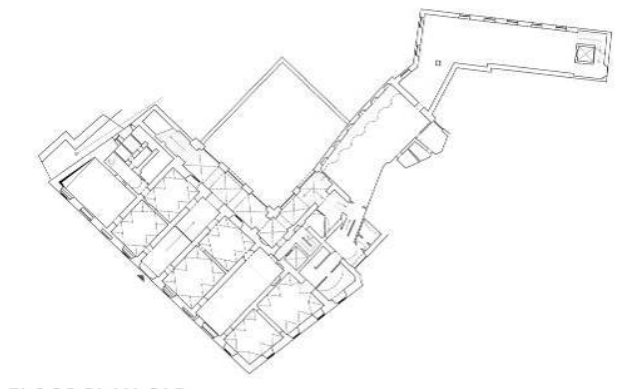

FLOOR PLAN CAD
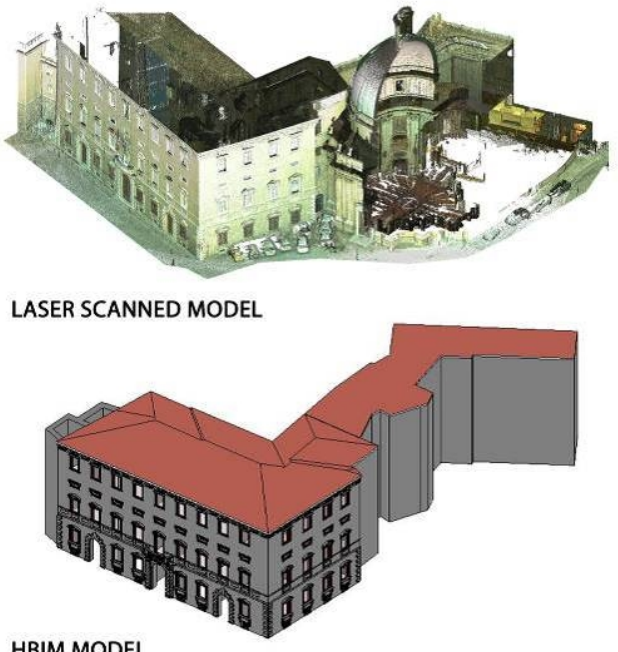

HBIM MODEL

Figure 2. Palazzo Ferretti: from cad to TLS and HBIM model

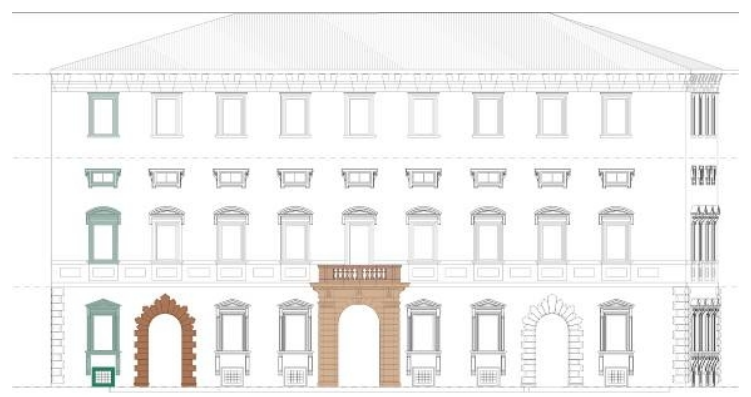

Figure 3. HBIM of Palazzo Ferretti, main façade: individuation of loadable families. In green the windows and in brown monumental entrances

However, the major phase has involved the moldings of main façades with the realization of loadable families (*.rfa) for which semantics and geometric constraints have been studied coherently with the practice of building and with the rules of the treaties. In fact, at this stage, the most significant results for Palazzo Ferretti concern the realization of parametric libraries for the external and internal building moldings.

All the windows, the door frames and boss, the triumphant balcony and all frames have been defined, with their basic elements and semantically connected thanks to the nomenclature of the parties and to the inherent structure in Revit (Figure 3). This is a robust nucleus of a typical ontology for a Renaissance building at different LOD (Figure 4).

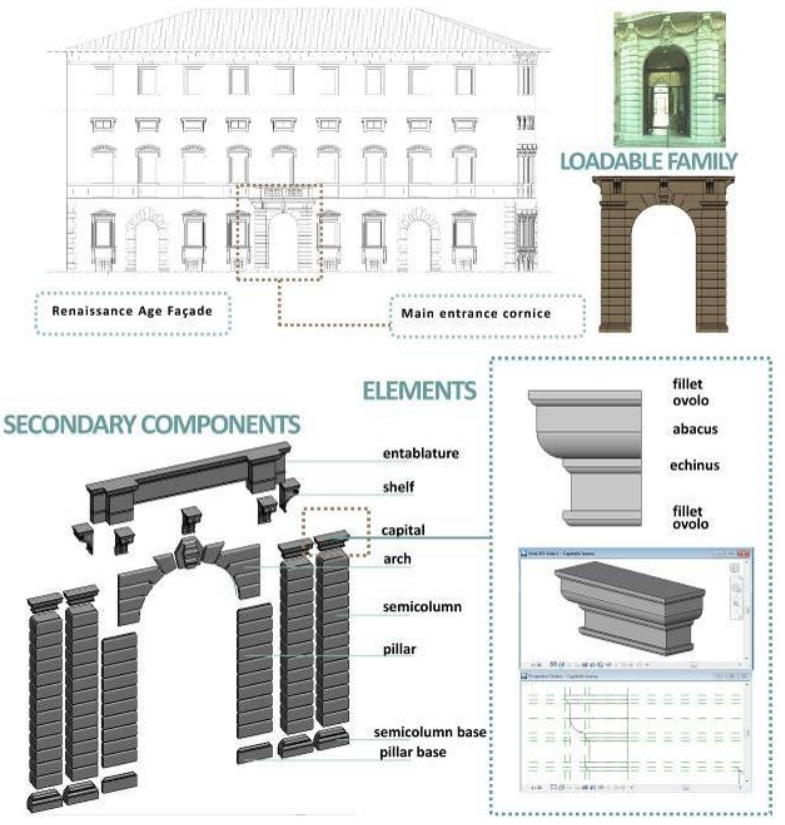

Figure 4. Semantic structure of a loadable family: bottom - up link from basic elements to *.rfa file.

The coherence within HBIM model and all sources was also assured by several documentation stages: for example an interesting step was the comparison between orthophotos from TLS point cloud and Structure from Motion (SfM) acquisition (Figure 5).

Specifically, in this paper the case study aims to validate a workflow able to handle all the information available in a hierarchically managed database using ontologies and taxonomies. This could be useful for readers if compared with the following case study. These ontologies and taxonomies represent the structuring of the data themselves and starting from some defined datasets they are linked to the threedimensional model in different ways. In the case of Palazzo Ferretti, we not only implemented datasets that represented composition and architectural elements of the building, but also other ones suitable for the description of Palazzo Ferretti as an art gallery. For this goal, a specific data enrichment was performed through Revit and using the shared parameters: we added to HBIM the archaeological evidences as specific instances referred to 3D models (with external or encapsulated links) and historical data.

Once the modelling of Palazzo Ferretti was completed, to enable a more complete and exhaustive data enrichment, we created families to identify various archaeological evidences in rooms of the Museum. The realization of loadable families, renamed "Archaeological Find" has gone through the creation of a generic cylindrical shape in order to ideally identify the finds within the modeled space. Once the "Archaeological Find" family has been obtained, this has been loaded into Palazzo Ferretti project through the "Family Editor" section.

Created parameters, linked to different thematic areas, could give different type of information referred on each single find: text, url and image (pdf 3D) (Figure 6).

Another following step regarded the question of interoperability. After having structured and made the connection between the model and the database we decided to carry out an export in ISO ifc format. The choice of this format 

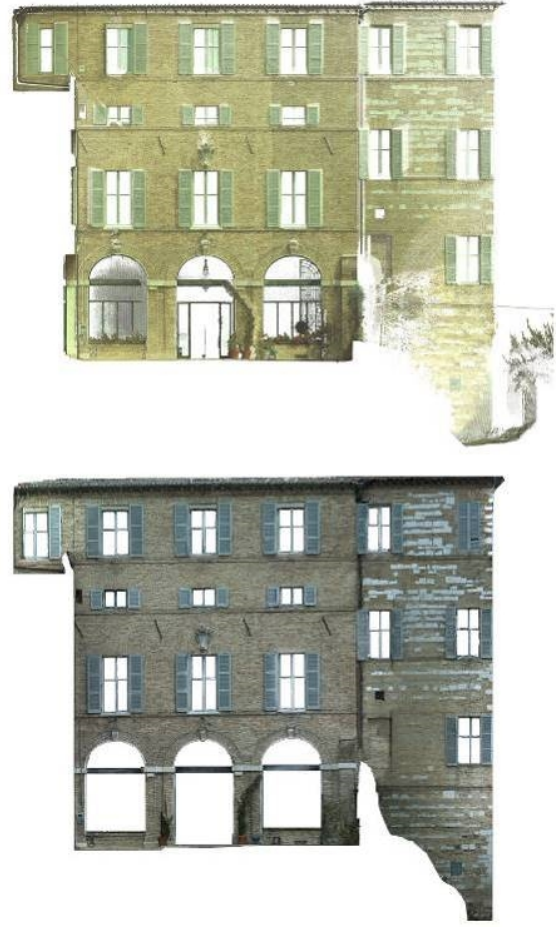

Figure 5. Comparison between orthophotos: TLS and SfM

seems the most logical considering that it is supported by many software available on the market.

After the export to IFC we checked the maintenance of the data structure and the compatibility with other descriptors. This will allow us to evaluate the interoperability and the ontological accuracy of the model exporting to IFC format and analyzing the output file to detect inconsistencies or data loss when moving from different work environments.

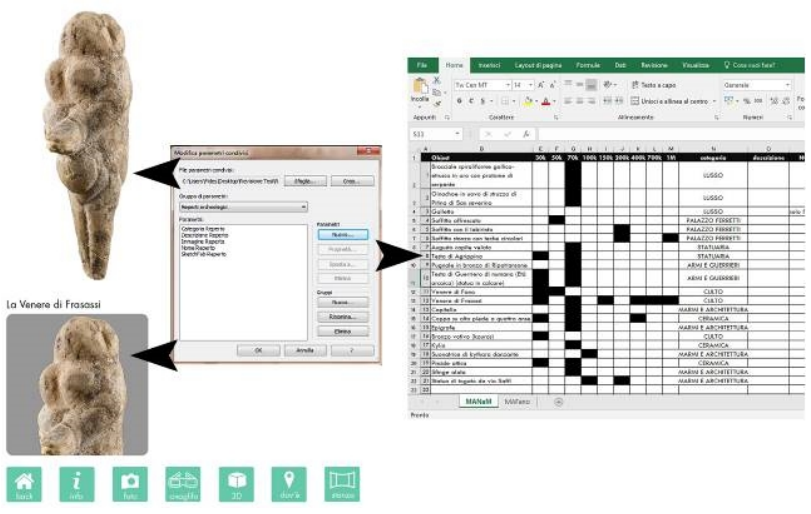

Figure 6. Shared parameters and information extracted for the archaeological evidence "La Venere di Frasassi"

\subsection{La Manzana Histórica de la Universidad Nacional del Litoral (MHUNL)}

"El edificio de la Manzana Histórica" of Universidad Nacional del Litoral is recently named National Historical Monument in Argentina because it was the first building for a public university in the whole country. The HBIM methodology was considered as a potential solution to its update, upgrade and valorisation. In Argentina, the main problem is that all the information is made up by a collection of individual and disconnected documents, dispersed in different places. This situation makes the access to the documentation difficult, also increases the time of research and causes errors in the decision making process. The current state of the information is the main cause of improper maintenance and incorrect protocols of preservation and restorations or modifications.

The implementation of several technological innovations has produced a series of new methodological and instrumental resources, which began to provide potential answers to the problem of the lack of information about the built environment related to cultural heritage.

This work describes a possible alternative for the creation and development of an information system that combines multidimensional data as shown in this case study (Figure 7). Aware that every heritage building has its own location characteristics and constructive architectural features that makes each one unique, it is necessary to find different strategies customized to the building under study; particular situations that determine the type of operations to be performed. (Figure 8).

To achieve a proper HBIM process we had the operational need to carry out a protocol that allows us to obtain a rapid assessment of the state of a heritage building in an efficient manner. This protocol is based in the APGAR ${ }^{1}$ score, a method to quickly summarize a concrete situation with multiples crosses of information and great uncertainty. We chose six common parameters to evaluate all heritage buildings; having into account that they are the most determining parameters to plan a work process related to the analysis of the buildings.

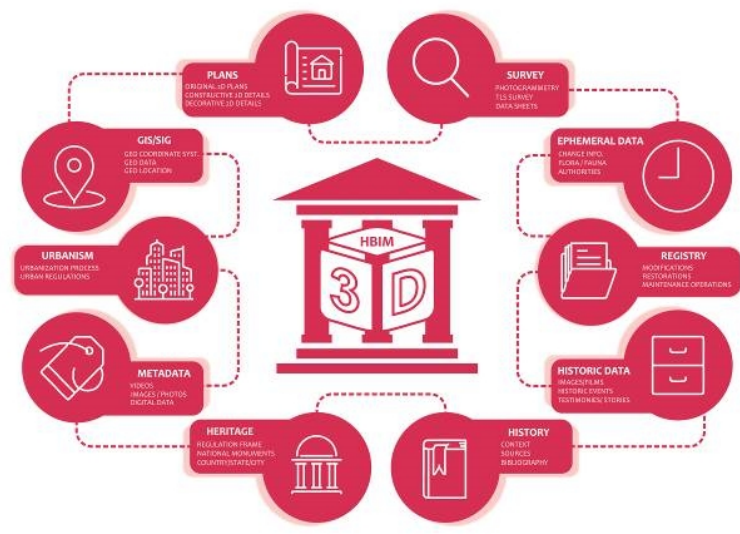

Figure 7. HBIM conceptual scheme

These parameters are evaluated based on a score table that values between 0 and 2; adding the sum of each parameter, we obtain the total valuation of the building under study. The range of assessment oscillates between 0 and 10 points, defining the maximum value obtained as the most favorable state of situation.

1 Lichtig, Will. Projects as Patients. What can we learn from the medical profession?. Will Lichtig. The American Institute of Architects, 2004. A Joint Project of AIA California Council Integrated Project Delivery Steering Committee AIA National Integrated Practice Discussion Group". Research and report by Jonathan Cohen, FAIA For more detailed background information on IPD, visit www.ipdca.net and refer to The Integrated Project Delivery Guide, jointly developed by the AIA's Integrated Practice Discussion Group and AIA California Council, and Integrated Project Delivery: A Working Definition, published by AIA California Council.

For information on existing project delivery methods, see the AIACC's Handbook on Project Delivery. More information can be read here: https://www.newyorker.com/magazine/2006/10/09/the-score 
In the case of MHUNL we got a score that allowed us to have a picture of the present situation and helped us to establish a proper path of activities to go along with the tasks we need to perform in the future. Although we had good sets of information about the architectural language and the construction system used in this building, but we do not have yet a trustable technical database. This protocol as tool of diagnosis allowed us to discover the main obstacles present at the location of the building, which helped us to plan the survey also anticipate possible future problems (Figure 9). Once determined the real situation of MHUNL, we were able to plan a photogrammetric and laser scanning survey only from the outside of the building, according to a general workflow (Figure 10).

Several technological equipment were used: a Leica C10 laser scanner for 12 scans of the exterior of the building; a drone Dji Inspire One Pro to obtain more accurate information of the top parts of the building and a digital camera Nikon 7500 to take photos of ornaments and specific decorations. Even though we do not have our own equipment or the necessary money to carry out this type of activities; we were able to perform a comprehensive survey campaign adequate to our possibilities, sharpening our critical thinking and management capacity to awaken the interest of local companies that helped us to go ahead with this endeavor (Figure 11).

The recognition that we are short of data to make a complete and accurate model of the entire building (Figure 12, Figure 13), push us to have a thinking strategy for decision making that harmonizes tradition and innovations. By streamlining the data obtained from different sources, grants us to lay the foundations of a work process based on broader view of HBIM methodologies, customized to a specific work environment as new method to manage multidimensional information efficiently.

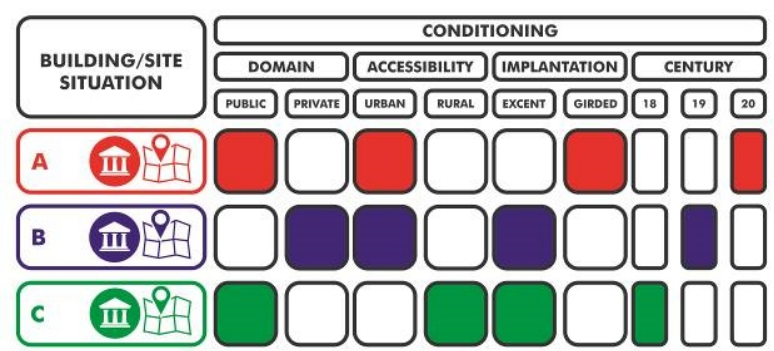

Figure 8. Situation Table

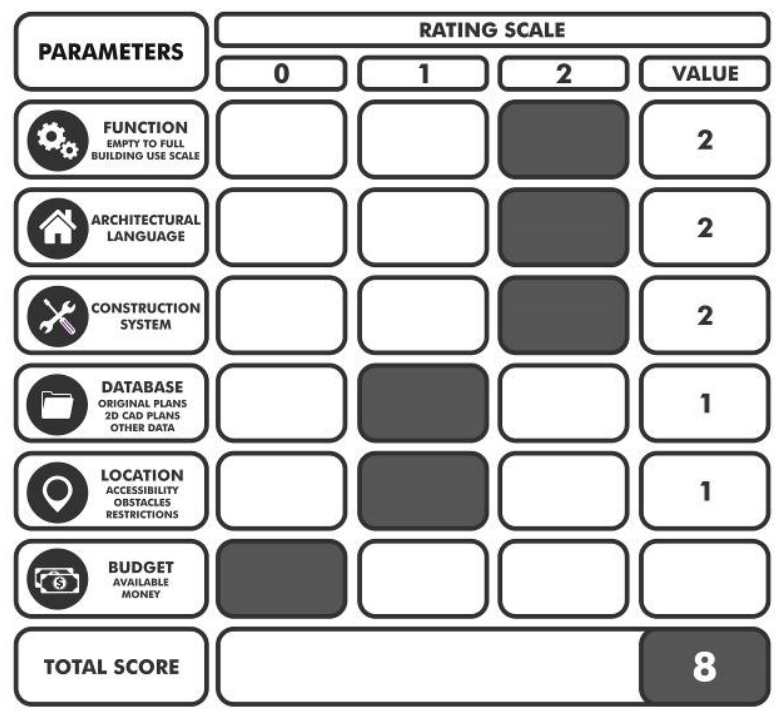

Figure 9. Protocol Table for Manzana Histórica Building.

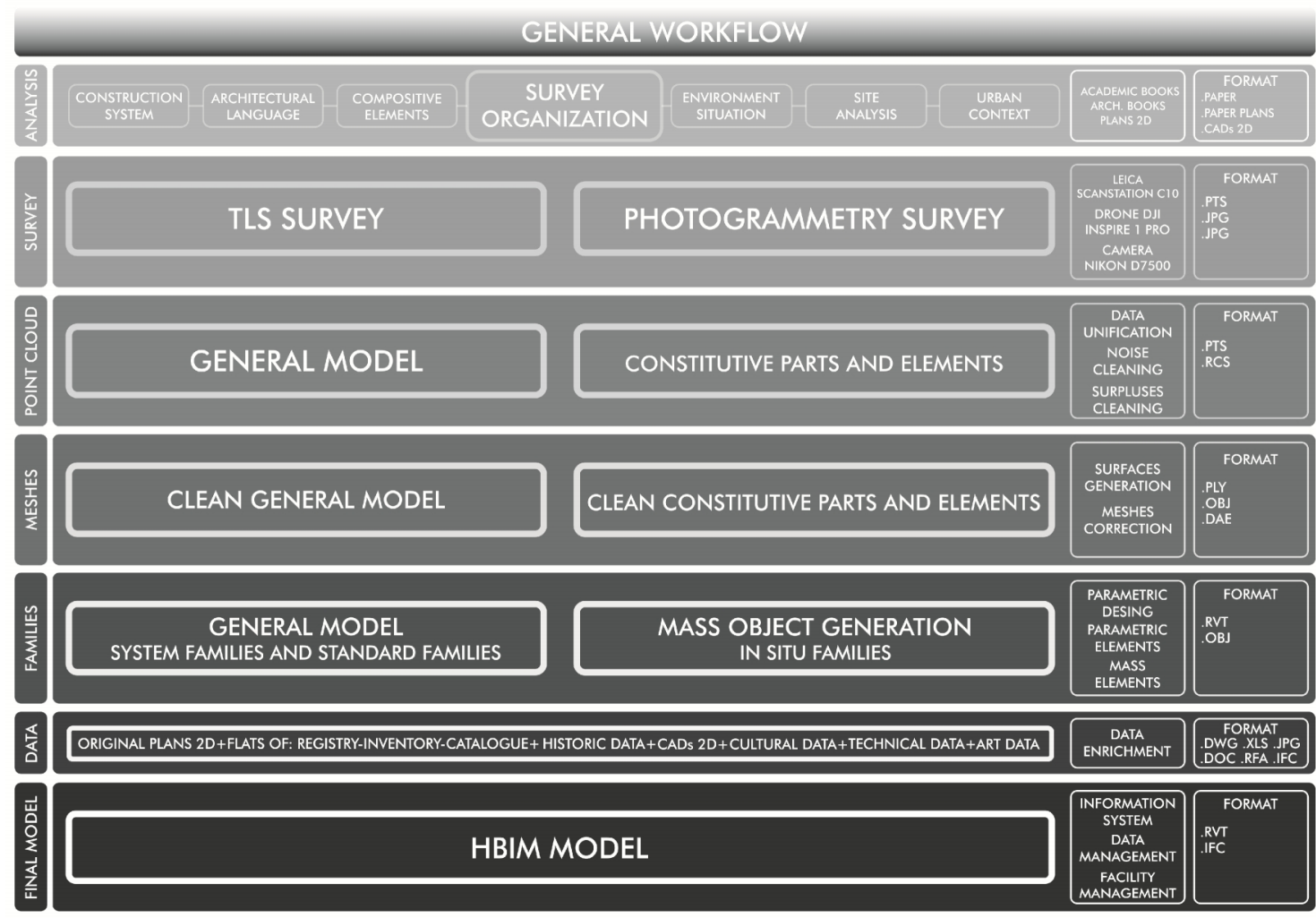

Figure 10. General workflow: from the analysis to HBIM final model 


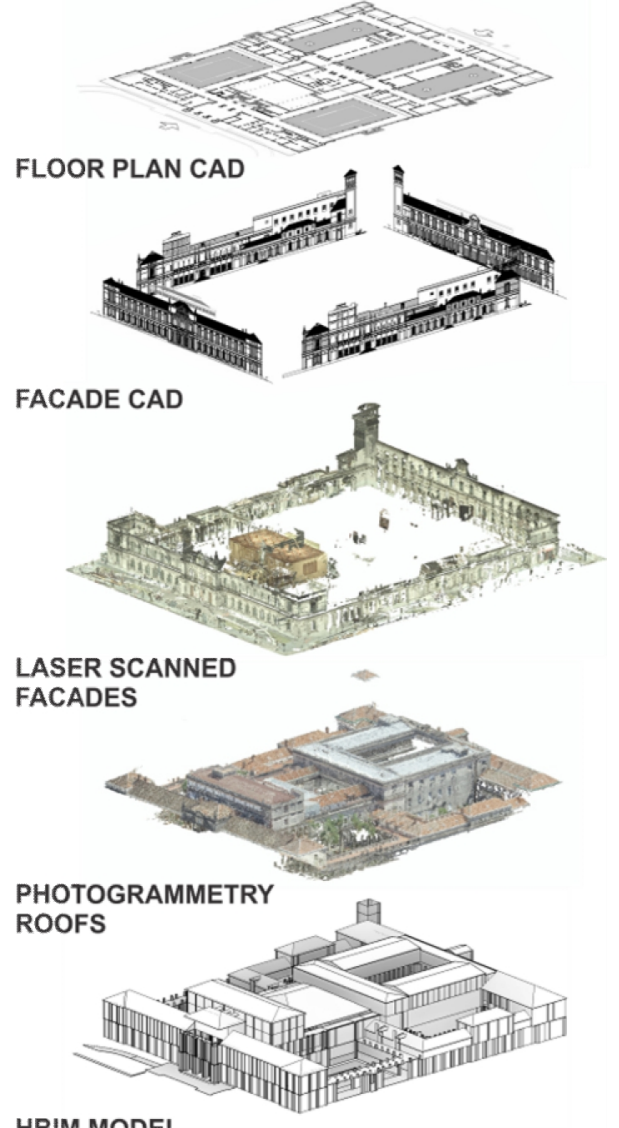

HBIM MODEL

Figure 11. Phases of the work

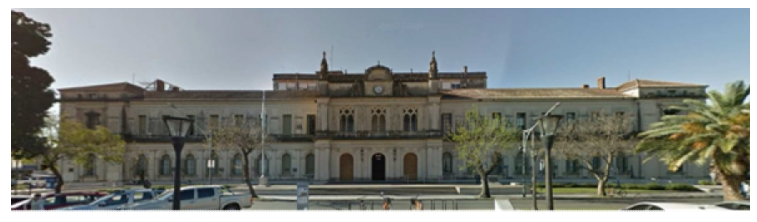

FACADE PHOTO
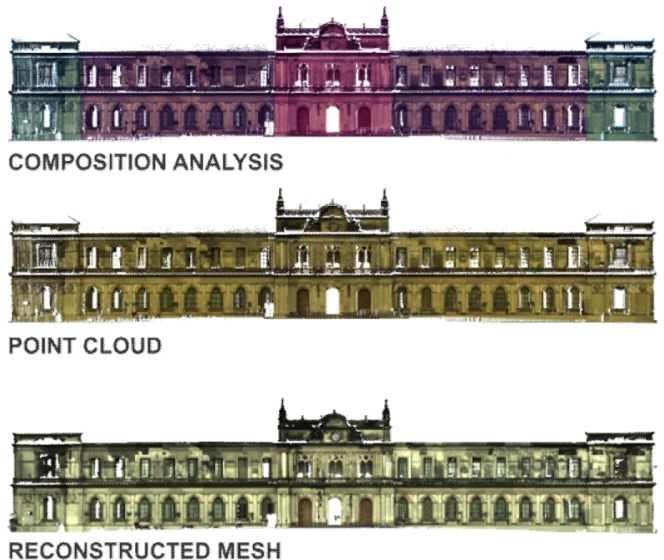

Figure 12 Phases to obtain ad accurate model: photogrammetric acquisition, façade composition analysis, point cloud and reconstructed mesh of Manzana Histórica

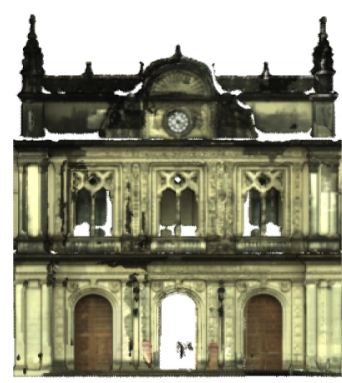

MAIN PART MESH

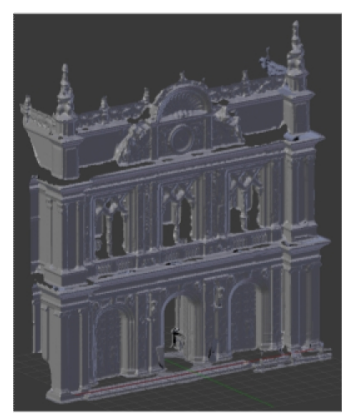

MESH SCULPTING

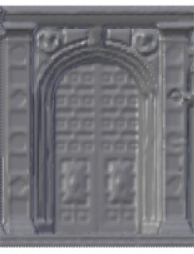

REPEATED ELEMENT

Figure 13. Workflow and mesh treatment of a part of Manzana Histórica main façade

\section{DISCUSSION AND RESULTS}

The comparison between the works presented here makes it possible to understand the effectiveness and the necessity of dissemination of suitable HBIMs, obtained by using appropriate research and conservation analysis tools. The use of different datasets, which orient the link between model and information, allows to represent and supply large quantities of specialized information for the knowledge of the historical heritage.

The main goal of the presented work is to focus on the potential solutions offered by the use of today information models, generated through the application of technological innovations tools that allows to capture the built environment. Achieving it will not only demonstrate the lack of precision of the documentation obtained through surveys based on traditional methods, also the potentiality of accessibility and the ability to 
incorporate information in a multidimensional digital model (Figure 14).

The importance of this proposal is the recognition of the relevant role that the administration, the organization and access to information are the main challenges in the processes of conservation, restoration and maintenance of heritage buildings (Figure 15).

The results obtained from the actual application of this methodology on the building of MHUNL are also discussed, and the implication of the application of this method, not only will allow the continuous incorporation of new information regarding the building, but also its permanent feedback and updating. The diffusion of similar practices could guarantee instantaneous accessibility through different types of electronic devices, and their preservation in an organized and hierarchical manner.

Another key aspect to be discussed is about LOD: obtaining standard shared procedures could facilitate HBIM development and diffusion in operating workflows. Interesting results arose from the evaluation of the different definitions, ranges and scales for LOD in America (100-500) and in Italy (A-G), also considering that the recently released UNI 11337 is expected to be scaled up at European level. Semantically structured data (ontologies) in both models allowed a complete management of the repositories and the dissemination of the methodology permit to validate the quality of the different levels of detail definition. Both BIM models are reliable as - built, corresponding respectively to the $\mathrm{F}$ grade, i.e. the restoration scale (Palazzo Ferretti) and to the LOD 500, used in heritage rehabilitation and maintenance projects for the Manzana Histórica. The elements of the models are represented in their entirety and have been associated with graphical and nongraphical information like technical documentation, such as floor plans, sections, elevations and details or external links.

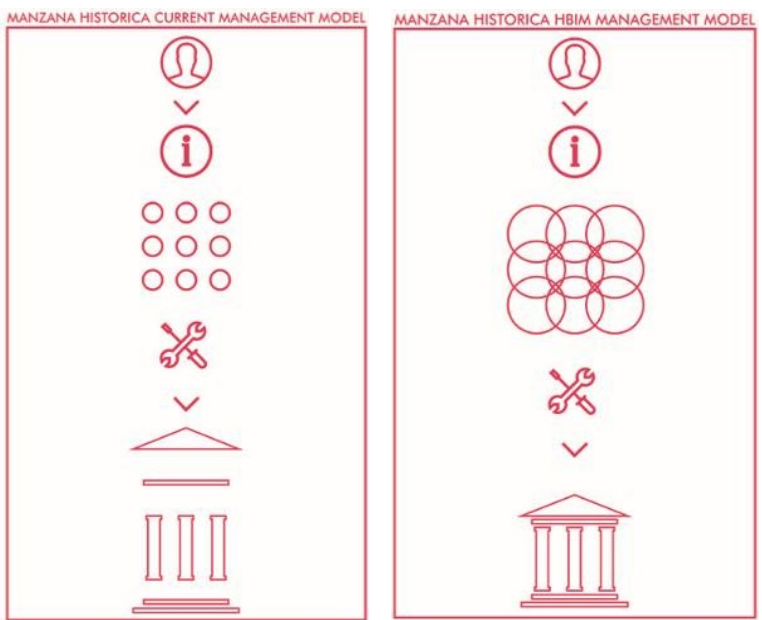

Figure 14. Comparison between current and HBIM management of the model

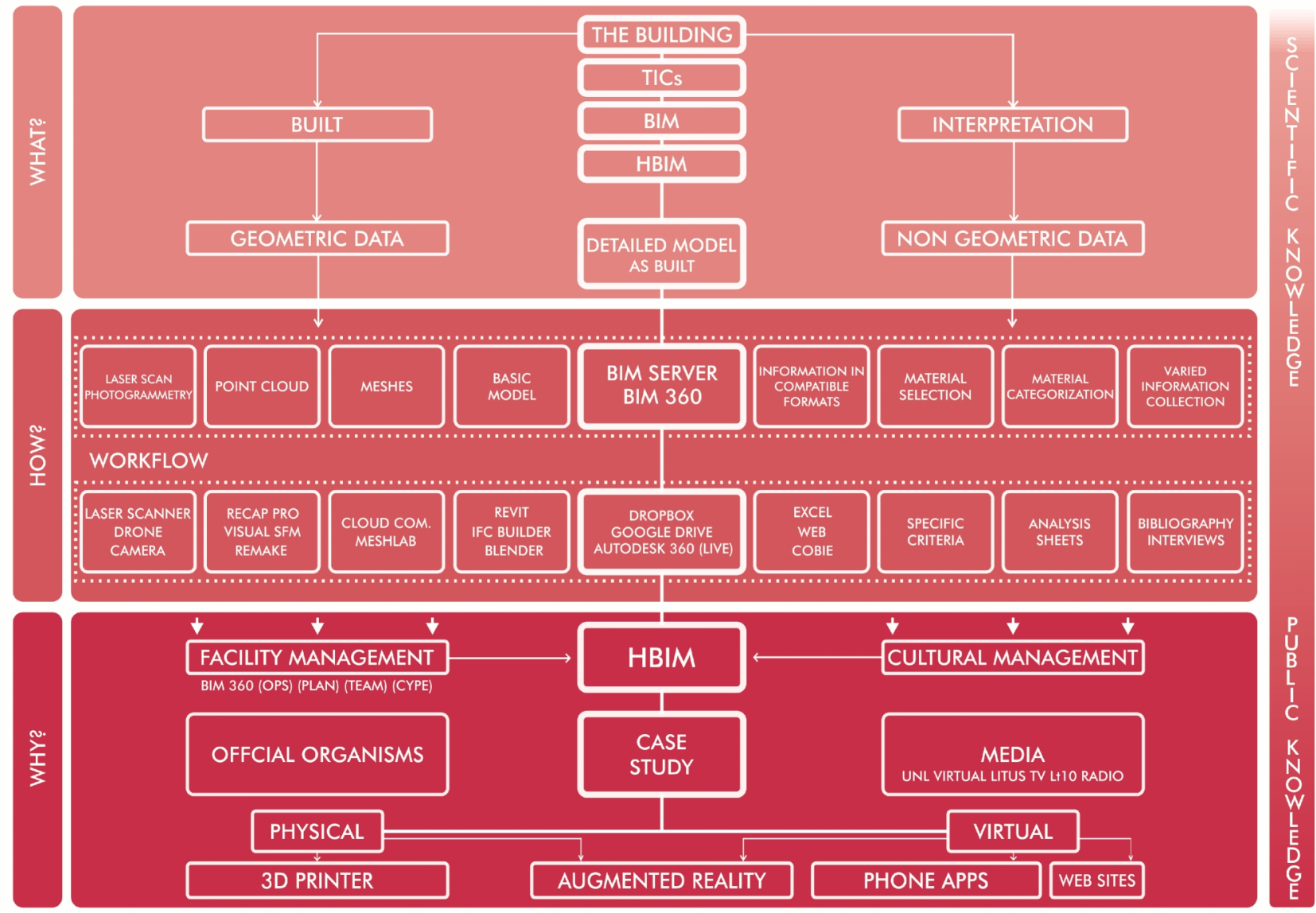

Figure 15. From a scientific to a public knowledge 


\section{CONCLUSIONS}

The analysis and assessment of the two presented procedures constitute a proper inquiry to enable the method extension to all historical buildings. Major challenges were expected in elimination of all those steps that cause lose efficiency and precision, but now we are able to observe that specific and useful improvements arise only merging theoretical and technical approaches.

We are able to conclude that the comparison between the cases study can validate a workflow that allow achieving a useful and proper data enrichment of each HBIM model. This is possible by implementing a database of functional metadata for an easy management and usability in Revit environment and in technical-professional context. The model separates itself from its canonical definition of simple representation, becoming a container of information, more or less useful. The flexibility of used methodology also allows to implement the model making it available at any time, with the opportunity to modify it by adding information. The model will be able to extend its knowledge and receive constant updates over time.

\section{ACKNOWLEDGEMENTS}

The authors want to acknowledge Nicoletta Frapiccini, the director of Palazzo Feretti's Gallery, and Prof. Paolo Clini for allowing the use of data survey. The acquisition phase was performed under its supervision by Romina Nespeca, Ludovico Ruggeri and technicians of Dicea Department. The modelling phase of Palazzo Ferretti was carried out in training and degree thesis by Fides Carosi, Valentina Carducci, Vincenzo Danzi, Marco Mattioli e Maria Laura Pierpaoli, relator Prof. Ramona Quattrini at the Polytechnic University of Marche.

The HBIM model of Manzana Histórica was performed by the students Maggiolo Gaston, Cristaldo German, Bernal Roberto, Ocampo Alejandra, who are completing their final career work in the Faculty of Architecture, Design and Urbanism of the National University of the Litoral Santa FE, Argentina. This case study is directed by Mg. Arq. Alejandro Moreira, Dr. Arq. Cecilia Parera.

\section{REFERENCES}

Apollonio, F.I., Basilissi, V., Callieri, M., Dellepiane, M., Gaiani, M., Ponchio, F., Rizzo, F., Rubino, A.R., Scopigno, R., Sobra', G., 2018. A 3D-centered information system for the documentation of a complex restoration intervention. J. Cult. Herit. 29 , 89-99.
https://doi.org/10.1016/J.CULHER.2017.07.010

Apollonio, F.I., Gaiani, M., Sun, Z., 2016. A Reality Integrated BIM for Architectural Heritage Conservation. Handb. Res. Emerg. Technol. Archit. Archaeol. Herit. 31.

Barazzetti, L., Banfi, F., Brumana, R., Previtali, M., 2015. Creation of Parametric BIM Objects from Point Clouds Using Nurbs. Photogramm. Rec. 30, 339-362.

COMMITTE, U., 2017. UNI 11337-4:2017 Edilizia e opere di ingegneria civile - Gestione digitale dei processi informativi delle costruzioni - Parte 4: Evoluzione e sviluppo informativo di modelli, elaborati e oggetti.

Davenport, T.H., Prusak, L., 2000. Working knowledge. Ubiquity 2000, 2-es. https://doi.org/10.1145/347634.348775

Garagnani, S., Manferdini, A.M., 2013. Parametric accuracy: Building Information Modeling process applied to the cultural heritage preservation, in: 3DArch2013, Conference Proceedings of The International Archives of the Photogrammetry, Remote Sensing and Spatial Information Sciences, Trento, Italy. Citeseer, pp. 87-92.

https://www.aiacontracts.org/contract-documents/19016project-bim-protocol [WWW Document], n.d.

Merschbrock, C., Munkvold, B.E., 2015. Effective digital collaboration in the construction industry - A case study of BIM deployment in a hospital construction project. Comput. Ind. 73, 1-7. https://doi.org/10.1016/J.COMPIND.2015.07.003

Quattrini, R., Malinverni, E.S., Clini, P., Nespeca, R., Orlietti, E., 2015. From tls to hbim. high quality semantically-aware $3 \mathrm{~d}$ modeling of complex architecture, in: International Archives of the Photogrammetry, Remote Sensing and Spatial Information Sciences - ISPRS Archives.

https://doi.org/10.5194/isprsarchives-XL-5-W4-367-2015

Quattrini, R., Pierdicca, R., Morbidoni, C., 2017. Knowledgebased data enrichment for HBIM: Exploring high-quality models using the semantic-web. J. Cult. Herit. 28. https://doi.org/10.1016/j.culher.2017.05.004

Schreyer, P., 2001. The OECD Productivity Manual: A Guide to the Measurement of Industry-Level and Aggregate Productivity. Int. Product. Monit. 2, 37-51.

Volk, R., Stengel, J., Schultmann, F., 2014. Building Information Modeling (BIM) for existing buildings - Literature review and future needs. Autom. Constr. 38, 109-127. 